\title{
Fabrication and characterization of PECVD silicon nitride for RF MEMS applications
}

\author{
H. U. Rahman ${ }^{1,2}$, B. C. Johnson ${ }^{3}$, J. C. Mccallum ${ }^{3}$, E. Gauja ${ }^{2}$, R. Ramer ${ }^{2}$ \\ ${ }^{I}$ The Department of Electrical Engineering, \\ College of Electrical and Mechanical Engineering \\ The National University of Sciences and Technology (NUST), Islamabad, Pakistan \\ hamood@ceme.nust.edu.pk \\ Tel: $+92-51-9278050$ \\ Fax: $+92-51-9278048$ \\ ${ }^{2}$ The School of Electrical Engineering and Telecommunications, \\ The University of New South Wales (UNSW), \\ Sydney, NSW 2052, Australia \\ ${ }^{3}$ Centre for Quantum Computer Technology, School of Physics \\ The University of Melbourne, \\ Parkville, Victoria 3010, Australia
}

\begin{abstract}
The structural, optical and electrical properties of plasma enhanced chemical vapor deposited silicon nitride layers are investigated, which have been used as a dielectric layer during RF MEMS fabrication. During growth, the gas ratio $\left(\mathrm{SiH}_{4} / \mathrm{NH}_{3}\right)$ is varied between $0.33-0.5$ and pressure is varied between $400-700 \mathrm{mTorr}$ while deposition time is kept constant. The results in the films show differing properties. The thicknesses of the resultant films are between 150 to $220 \mathrm{~nm}$ with different gas flow ratios and pressures whereas the deposition time was kept constant. A Bruggeman effective medium approximation is utilized to model the refractive index of the films. Reflectance measurements were carried out in the range of $210-250 \mathrm{~nm}$. The refractive indexes of the films varied between 1.79 to 2.03 , with a dielectric constant varying from 6.66 to 7.22. Capacitance voltage measurements yield a fixed dielectric charge value in the low- $10^{12} \mathrm{~cm}^{-2}$ while a breakdown voltage of $915 \mathrm{~V} / \mu \mathrm{m}$ is achieved for films grown at the lowest gas ratio and pressure. The quality of $\mathrm{Si} / \mathrm{Si}_{\mathrm{x}} \mathrm{N}_{\mathrm{y}}$ interface is also considered.
\end{abstract}

Index Terms: RF MEMS, PECVD, silicon nitride, series switch

\section{Introduction}

Radio frequency micro electromechanical systems (RF MEMS) based switches show great potential for use in mobile and satellite communication and can be integrated on chip with driving electronics (Mansour et.al. 2003). Silicon nitride films are commonly used for MEMS due to its hardness, chemical inertness and good electrical insulation properties (Hines et. al. 1995; Bustillo et. al. 1998; Daldosso et. al. 2004 ). Various methods can be used to produce $\mathrm{Si}_{\mathrm{x}} \mathrm{N}_{\mathrm{y}}$ films; they include thermal chemical vapor deposition (CVD), thermal nitridation of silicon, plasma enhanced chemical vapor deposition (PECVD), ion implantation, ion beam deposition, pulse laser ablation, reactive evaporation and reactive sputtering (Olson 2002). In addition to switches a number of other $\mathrm{RF}$ components can use $\mathrm{Si}_{\mathrm{x}} \mathrm{N}_{\mathrm{y}}$ as a dielectric layer including inductors, capacitors, phase shifters and surface acoustic wave devices. The optical and electrical properties of $\mathrm{Si}_{\mathrm{x}} \mathrm{N}_{\mathrm{y}}$ layers must be optimized for each type of device.

In addition to RF MEMS devices, $\mathrm{Si}_{\mathrm{x}} \mathrm{N}_{\mathrm{y}}$ films have found application as oxidation masks, protection and passivation barrier layers, etch stop layers and inter-level insulators for isolation 
in the semiconductor industry (Sanders et. al.1997; Xu et. al. 2003). A near stoichiometric silicon nitride, deposited at low temperature is used for these applications and has a high breakdown voltage and low interface trap density (Daldosso et. al. 2004). $\mathrm{Si}_{\mathrm{x}} \mathrm{N}_{\mathrm{y}}$ is also a candidate to replace $\mathrm{SiO}_{2}$ in ultra-thin dielectric structures since $\mathrm{Si}_{\mathrm{x}} \mathrm{N}_{\mathrm{y}}$ has a higher dielectric constant and exhibits better performance as a diffusion barrier than $\mathrm{SiO}_{2}$ (Dueñas et. al. 1997). Despite their widespread application, there is only a few reported works in the literature that deal with the electrical properties of the $\mathrm{Si} / \mathrm{Si}_{\mathrm{x}} \mathrm{N}_{\mathrm{y}}$ interface (Dueñas et. al. 1997; Stesmans 1989; Garcia et. al. 1998; Gong et. al. 2010 ).

Here, the deposition of $\mathrm{Si}_{\mathrm{x}} \mathrm{N}_{\mathrm{y}}$ by PECVD is studied for application as a dielectric layer in RF MEMS switches. It is shown that the properties of the layers can be customized by varying the deposition conditions such as the gas flow ratio and the chamber pressure. Reflectance measurements were used to measure the quality and stoichiometry of the film. Capacitancevoltage $(\mathrm{C}-\mathrm{V})$ and deep level transient spectroscopy (DLTS) measurements are also used to quantify the interface state density. Finally, the fabrication process for RF MEMS switches indicating the use of $\mathrm{Si}_{\mathrm{x}} \mathrm{N}_{\mathrm{y}}$ as a dielectric layer is discussed.

\section{Experimental Procedure}

$\mathrm{Si}_{\mathrm{x}} \mathrm{N}_{\mathrm{y}}$ films were grown on 2 inch polished $\mathrm{n}$ type, p-doped (100) silicon wafers with a resistivity of $5 \Omega . \mathrm{cm}$ using a parallel plate PECVD reactor (VACUTEC-1500 plasma system). A schematic diagram of the PECVD apparatus is shown in fig. 1. The diameter of the PECVD reactor chamber is $305 \mathrm{~mm}$ and the diameter of the shower head through which the gases enter is $260 \mathrm{~mm}$ with a RF frequency of 13.56MHz. A gas mixture of $\mathrm{SiH}_{4} / \mathrm{NH}_{3}$ was introduced into the reaction chamber through a shower head in the upper electrode for $\mathrm{Si}_{\mathrm{x}} \mathrm{N}_{\mathrm{y}}$ deposition under different processing conditions.

The $\mathrm{SiH}_{4} / \mathrm{NH}_{3}$ ratio was varied from 0.33 to 0.5 while the pressure was varied from 400700mTorr. The wafer temperature during growth was measured from a lower electrode and was kept constant at $300^{\circ} \mathrm{C}$. Other parameters such as power and deposition time were kept constant to $75 \mathrm{~W}$ and $8 \mathrm{~min}$, respectively. The experimental deposition parameters are shown in table 1 . These parameters resulted in a stoichiometric ratio of $\mathrm{Si}_{\mathrm{x}} \mathrm{N}_{\mathrm{y}}$ with $x=3.03$ and $y=4$ as determined with ellipsometry measurements.

Table 1. Deposition conditions for the $\mathrm{Si}_{\mathrm{x}} \mathrm{N}_{\mathrm{y}}$ films formed under three processing routines

\begin{tabular}{ll}
\hline Substrate type & $\mathrm{Si}$ n-type $(100) 5 \Omega . \mathrm{cm}$ \\
Substrate temperature & $300{ }^{\circ} \mathrm{C}$ \\
RF power & $75 \mathrm{~W}$ \\
Time & $8 \mathrm{~min}$ \\
Pressure & $400-700 \mathrm{mTorr}^{-}$ \\
Ratio-1 & $\left(\mathrm{SiH}_{4} / \mathrm{NH}_{3}\right)=0.333$ \\
Ratio-2 & $\left(\mathrm{SiH}_{4} / \mathrm{NH}_{3}\right)=0.375$ \\
Ratoi-3 & $\left(\mathrm{SiH}_{4} / \mathrm{NH}_{3}\right)=0.5$ \\
\hline
\end{tabular}

After deposition, various properties of the films were measured. They include, the refractive index, growth rate, surface roughness $\left(\mathrm{R}_{\mathrm{a}}\right)$, breakdown voltage, dielectric constant and flatband voltage.

Firstly, reflectance measurements were performed using a CARY 5G photospectrometer. The measured data was then compared with modeled reflectance data for the $\mathrm{Si}_{\mathrm{x}} \mathrm{N}_{\mathrm{y}}$ films, using a least squares analysis. The modeled reflectance data was simulated using WVASE ellipsometric software which implements the Fresnel thin film equations for reflectance.

The input parameters for the simulated reflectance curves are the film thickness (measured using a DEKTAK profilometer and AFM) and the optical parameters refractive index $(n)$ and extinction coefficients $(k)$, which were to be determined.

The refractive indexes and extinction coefficients of the films were determined by fitting the modeled and measured reflectance data, using a Bruggeman effective medium model (Niklasson et. al. 1981). The modeled reflectance data consists of known refractive index data for silicon nitride, amorphous-Si (a-Si) and voids of the Bruggeman model. The measured reflectance data was used as the fitting parameter with the modeled data which allowed the determination of the refractive indexes of our films. It should be noted that the use of $\mathrm{Si}_{\mathrm{x}} \mathrm{N}_{\mathrm{y}}$, a-Si and voids in the Bruggeman effective medium model allows us to vary the refractive index of our fit, but does not imply the actual presence of a-Si and voids in the films.

Metal-insulator-semiconductor (MIS) structures were fabricated with $250 \mu \mathrm{m}$ diameter $\mathrm{Al}$ dots 
evaporated on the $\mathrm{Si}_{\mathrm{x}} \mathrm{N}_{\mathrm{y}}$ films for current-voltage (I-V), capacitance-voltage $(\mathrm{C}-\mathrm{V})$ measurements and deep level transient spectroscopy (DLTS). I$\mathrm{V}$ measurements were performed at room temperature with SONY Tektronix-370 curve tracer high current source measurement unit. The supply voltages from $-40 \mathrm{~V}$ to $+400 \mathrm{~V}$ were used to determine the electric breakdown field. C-V and deep level transient spectroscopy (DLTS) measurements were performed at room temperature on a SULA Technologies DLTS system. C-V scans were performed from inversion to accumulation at a rate of $0.005 \mathrm{~V} / \mathrm{s}$ to ensure the capacitor was in equilibrium throughout the measurement. DLTS was also performed to characterize the $\mathrm{Si}-\mathrm{Si}_{\mathrm{x}} \mathrm{N}_{\mathrm{y}}$ interface. The DLTS spectra were formed by a standard box-car method with a $1 \mathrm{MHz}$ AC probe frequency.

\section{Results and Discussion}

\subsection{Deposition Rate vs. Pressure}

Fig. 2 shows the variation in deposition rate as a function of the gas ratios and pressures listed in table 1. As the gas pressure increases the deposition rate increases. As the gas ratio $\mathrm{SiH}_{4} / \mathrm{NH}_{3}$ increases the deposition rate also increases. The deposition time was kept constant so that film thicknesses could be controlled. The deposition rate is almost linear with the change in pressure.

\subsection{Reflectance Measurements}

Fig. 3 shows the refractive index, $n$, variation at $\lambda=633 \mathrm{~nm}$ as a function of gas ratio and pressure. There is an increase with gas pressure with a peak in refractive index at 600mTorr independent of the gas ratio used. The refractive indexes for films grown in gas Ratio-3 are noticeably higher than the other films. It is possible to customize the refractive index of the film by managing deposition conditions such as pressure and gas flow ratios (Daldosso et. al. 2004).

\subsection{Pressure vs. Surface Roughness}

The surface roughness of the films was assessed by measuring roughness parameters, $R_{a, r m s}$ using atomic force microscopy (AFM). $R_{a, r m s}$ is defined as the root mean square average of the height $(H)$ deviations taken from the mean data plane. It is expressed as

$$
R_{a, r m s}=\sqrt{\frac{1}{N} \sum_{i=1}^{N}\left(H_{i}-\bar{H}\right)^{2}}
$$

where $N$ is the number of points with the given area, $H_{i}$ is the current height value and $\bar{H}$ is the average of the height values within the plane.

In fig. 4 , it is also observed that a high gas ratio results in a relatively high surface roughness which increases with gas pressure.

The other two gas ratios considered have a surface roughness less than half of Ratio-3. The dependence on gas pressure for these films is weaker but tend to suggest that a maximum surface roughness is achieved for 500 and 600mTorr for Ratio-1 and 2, respectively.

\subsection{C-V Measurements}

A typical C-V curve is shown in fig. 5 for a sample grown in a ambient pressure of 400 mTorr with gas Ratio-3. The voltage is pulsed from $0 \mathrm{~V}$ to $-13 \mathrm{~V}$ at the start of the measurement. This results in driving the MIS into deep depletion. Some time is needed for the minority carrier to form and thus to reach equilibrium. This behavior is signified by the slow increase in capacitance up to the inversion capacitance which is reached at about $-11 \mathrm{~V}$. The scan rate was chosen to be $0.01 \mathrm{~V} / \mathrm{s}$ to minimize this.

The flatband voltage $\left(\mathrm{V}_{\mathrm{fb}}\right)$ for the $\mathrm{C}-\mathrm{V}$ curve shown in fig. 5 was $-9.2 \mathrm{~V}$. We estimate the fixed dielectric charge to be in the low $-10^{12} \mathrm{~cm}^{-2}$. Within experimental error, there is no change in fixed dielectric charge with the processing parameters considered here. The dielectric constant of this film was determined to be 6.7.

The variation in dielectric constant for the other films is shown in fig. 6. Generally, the dielectric constant decreases as the gas ratio increases. However, at a pressure of $600 \mathrm{mT}$ Torr the opposite is true. This may have some correlation with refractive index variation at this pressure and requires further investigation.

Fig. 7 shows the DLTS signal for a $\mathrm{Si} / \mathrm{Si}_{3} \mathrm{~N}_{4}$ interface where the $\mathrm{Si}_{3} \mathrm{~N}_{4}$ film was grown at a pressure 400 mTorr with gas Ratio-3. A peak is 
observed at low temperatures which is due to the $\mathrm{Si}=\mathrm{Si}_{3}$ defect $\left(P_{b o}\right)$ (Stesmans 1989).

This defect $P_{b o}$ is the surface Si dangling bond pointing out of the $\mathrm{Si}$ surface which is equivalent to the $P_{b 0}$ defect in the $\mathrm{Si} / \mathrm{SiO}_{2}$ system. The defect was found to have a trap energy of $0.23 \mathrm{eV}$ and a capture cross section of $\sigma=1.4 \times 10^{-13} \mathrm{~cm}^{2}$. The concentration of this defect was $4 \times 10^{12} / \mathrm{cm}^{2}$. Compared to a standard $\mathrm{Si} / \mathrm{SiO}_{2}$ interface this defect density is considerable. A prominent peak just above room temperature was found to dominate the spectra. This peak is due to generation-recombination processes. In general, the quality of the $\mathrm{Si} / \mathrm{Si}_{3} \mathrm{~N}_{4}$ interface did not vary significantly with the processing parameters considered here.

\subsection{I-V Measurements}

The breakdown voltage for the MIS capacitors is shown in fig. 8 as a function of the processing parameters. One can notice that the highest breakdown voltages are achieved with the lowest deposition pressures. This is despite the fact that the thickness of the films increases with gas pressure and so should provide more robust properties. The highest breakdown voltage is $915 \mathrm{~V} / \mu \mathrm{m}$ which was achieved with a film grown with Ratio-1 at a pressure of $400 \mathrm{mT}$ Torr. For Ratio-2 a similar trend was observed for the breakdown voltage.

As could be expected from the gas ratios, Ratio-3 has shown the highest excess Silicon and in turn has shown a low breakdown voltage of $410 \mathrm{~V} / \mu \mathrm{m}$. The lower breakdown voltage trend may be related to the high surface roughness of the film as indicated above. Silicon nitride films were deposited at a temperature of $300^{\circ} \mathrm{C}$. Fig. 8 shows that use of silane and ammonia exhibits a variable trend in the breakdown voltage.

As the concentration of silane increases the breakdown strength decreases to $308 \mathrm{~V} / \mu \mathrm{m}$ due to increase in conductivity. The same trend has also been reported by (Khaliq et. al. 1988).

\section{$4 \quad$ Fabrication}

The fabrication of the RF MEMS switch has been done by using silicon nitride as a dielectric layer. The detailed fabrication process along with its release method has been explained in (Rahman et. al 2010). However, main mask layers of the fabrication process are explained below.

The fabrication process is a six mask all metal process. DC bias lines and actuation pad are defined by evaporating $0.04 \mu \mathrm{m}$ layer of $\mathrm{Cr}$. This layer is then patterned with mask one. A $0.75 \mu \mathrm{m}$ thick layer of silicon nitride is deposited as insulator/dielectric layer using plasma enhanced chemical vapor deposition (PECVD) and patterned with mask two. Since the switch is made of metal, a dielectric layer is needed to prevent direct DC contact between the metal cantilever bridge and the actuation pad. The CPW lines are defined by evaporating/RF sputtering of $0.04 / 1.0 \mu \mathrm{m}$ thick layer of $\mathrm{Cr} / \mathrm{Au}$ and patterned with mask three. $\mathrm{Cr}$ has been used as an adhesion layer between the $\mathrm{Au}$ and substrate. Then a $2.5 \mu \mathrm{m}$ thick layer of photoresist (AZ-6632) is deposited as sacrificial layer and patterned for anchor and dimple with mask four and five respectively. This is followed by a $1.5 \mu \mathrm{m}$ thick layer of RF sputtered Au which is patterned with mask six to form the cantilever beam. Finally, the bridge structure is released by using a dry release process. Fig. 9 shows the fabricated switch indicating the $\mathrm{Si}_{\mathrm{x}} \mathrm{N}_{\mathrm{y}}$ layer used as a dielectric layer.

The details of the fabricated switch and RF performance have been discussed in (Rahman et. al. 2010). The fabricated switch actuated at a voltage of $23 \mathrm{~V}$. The isolation of the switches is better than $27 \mathrm{~dB}$ up to $40 \mathrm{GHz}$. The return loss is better than $18 \mathrm{~dB}$ and the insertion loss is $1.3 \mathrm{~dB}$ respectively, for all frequency band of interest. Using the same fabrication process several other novel RF MEMS switch designs have also been fabricated.

\section{Conclusion}

The deposition and properties of PECVD silicon nitride films have been studied as a part of the establishment of the fabrication process for RF MEMS devices. The properties of silicon nitride films were related to process conditions and substrate material. The increase in deposition rate was linear with change in pressure however, little affected by $\mathrm{SiH}_{4} / \mathrm{NH}_{3}$ flow ratios. The reflectance measurements indicated the changes in refractive index of the films. The refractive indexes of the films increased with an increase in gas pressure however, it decreased above $600 \mathrm{mT}$ orr gas pressure. The variation in refractive indexes can be noted with variation in 
gas ratios. The surface roughness of the films changed with increase in pressure and change in $\mathrm{SiH}_{4} / \mathrm{NH}_{3}$ ratios. However, in case of Ratio-1 and Ratio-2 surface roughness improved above 600mTorr gas pressure. C-V and DLTS measurements were used to quantify the interface state density. A peak was observed at low temperature which was due to $\mathrm{Si}: \mathrm{Si}_{3}$ defect. The defect was found to have trap energy of $0.23 \mathrm{eV}$ and captured cross section area of $1.4 \times 10^{-13} \mathrm{~cm}^{2}$ with concentration of defect to be $4 \times 10^{12} / \mathrm{cm}^{2}$. The highest breakdown voltage is $915 \mathrm{~V} / \mu \mathrm{m}$ which was achieved with a film grown with Ratio-1 at a pressure of $400 \mathrm{~m}$ Torr. The breakdown voltage decreased as the pressure increased. Similar trend was observed for the breakdown voltage in case of Ratio- 2 and 3 . The lower breakdown voltage trend may be related to the high surface roughness of the film however, use of silane and ammonia also exhibits a variable trend in the breakdown voltage. The application of silicon nitride film as a dielectric layer has been demonstrated in fabrication of RF MEMS series switch exhibiting satisfactory RF performance.

\section{References}

Mansour R. R., Bakri-Kaseem M., Daneshmand M., Messiha N. (2003) RF MEMS devices. In: Proceedings of Intl. Conf. on MEMS, NANO and Smart Systems (ICMENS 2003), pp 103-107

Hines J. H., Malocha D. C. et. al. (1995) Deposition parameters studies and surface acoustic wave characterisation of PECVD silicon nitride films on lithium niobate. In: Proceedings of IEEE Trans. on Ultrasonics, Ferroelectrics and Frequency Control, Vol. 42, No. 3, pp 397-402

Bustillo J. M., Howe R. T., Muller R. S. (1998) Surface micromaching for microelectromechanical systems. In: Proceedings of IEEE Vol. 86, No. 8, pp 1552-1574

Daldosso N., Melchiorri M., Riboli F. et.al. (2004) Fabrication and optical characterization of thin two-dimentional $\mathrm{Si}_{3} \mathrm{~N}_{4}$ waveguides. J Mat. Sci. Semicond. Processing, Vol. 7, Issues 4-6, pp 453-458, doi:10.1016/j.mssp.2004.09.023

Olson J. M., (2002) Analysis of LPCVD process conditions for the deposition of low stressed silicon nitride. Part I: Preliminary LPCVD experiments. J. of Mat. Sci. Semicond.
Processing Vol. 5, Issue 1, pp 51-60, doi:10.1016/S1369-8001(02)00058-6

Sanders T. J., Caraway E. L., Hall C. J. et.al. (1997) Silicon nitride deposition process for low cost microelectronics applications. In: Proceedings of the $12^{\text {th }}$ Bien. Univ./Govern./Ind. Microel. Symp. pp 173176

Xu G., Jin P., Tazawa M., Yoshimura K. (2003) Optical investigation of silicon nitride thin films deposited by r.f. magnetron sputtering. J Thin solid Films. Vol. 425, Issues 1-2, pp 196-202, doi:10.1016/S00406090(02)01089-1

Daldosso N., Melchiorri M., Riboli F., et.al. (2004) Fabrication and optical characterization of thin two dimentional Si3N4 waveguides. J Mat. Sci. in Semicond. Vol. 7, Issues 4-6, pp. 453-458, doi:10.1016/j.mssp.2004.09.023

Dueñas S., Pelaez R., Castan E., Pinacho R., Quintanilla L., Barbolla J., Martil I. and Gonzalez-Diaz G. (1997) Experimental observation of conductance transients in $\mathrm{Al} / \mathrm{SiN}_{\mathrm{x}}: \mathrm{H} / \mathrm{Si}$ metal-insulatorsemiconductor structures. Appl. Phys. Lett. 71, Issue 6, pp 826-828, doi: $10.1063 / 1.119658$

Stesmans A. (1989) The $\mathrm{Si}=\mathrm{Si} 3$ defect at various $111 \mathrm{si} / \mathrm{SiO} 2$ and $111 \mathrm{Si} / \mathrm{Si} 3 \mathrm{~N} 4$ interfaces. J Semicond. Sci. Technol. Vol. 4, No. 12, pp 1000-1011, doi: 10.1088/02681242/4/12/005

Garcia S., Martil I., Gonzalez Diaz G., Castan E., Duenas S. and Fernandez M. (1998) Deposition of $\mathrm{SiN}_{\mathrm{x}}: \mathrm{H}$ thin films by the electron cyclotron resonance and its application to $\mathrm{Al} / \mathrm{SiN}_{\mathrm{x}}: \mathrm{H} / \mathrm{Si}$ structures. $\mathrm{J}$ Appl. Phys., Vol. 83, pp 332-338, doi: $10.1063 / 1.366713$

Gong C. et. al. (2010) A deep-level transient spectroscopy study of silicon interface states using different nitride surface passivation scheme. Appl. Phy. Lett. 96, pp 1030507-1-103507-3, doi: $10.1063 / 1.3358140$

Niklasson G. A., Granqvist C. G., Hunderi O. (1981) Effective medium models for the optical properties of inhomogeneous materials. J Applied optics, Vol. 20, Issue 1, pp 26-30, doi: 10.1364/AO.20.000026

Khaliq M.A., Shams Q. A., Brown W. D., Naseem H. A. (1988) Physical properties of memory quality PECVD silicon nitride. J Electronic materials, Vol. 17, No. 5, pp 355-359, doi: 10.1007/BF02652118 
Rahman H. U., Chan K. Y. and Ramer R. (2010) Cantilever beam designs for RF MEMS switches. J Micromech. Microeng., Vol. 20, No. 7(075042), pp 12, doi: $10.1088 / 0960-1317 / 20 / 7 / 075042$

\section{List of Figures}

Fig. 1 Schematic diagram of the PECVD apparatus Fig. 2 Deposition rate as a function of pressure and gas flow ratios

Fig. 3 The dependence of the refractive index at $633 \mathrm{~nm}$ on the various deposition routines

Fig. 4 Surface roughness of silicon nitride film versus pressure

Fig. 5 Room temperature $\mathrm{C}-\mathrm{V}$ curve for a MIS capacitor with a $\mathrm{Si}_{\mathrm{x}} \mathrm{N}_{\mathrm{y}}$ film grown at a pressure 400mTorr with gas Ratio-3

Fig. 6 The dielectric constant determined from both thickness and $\mathrm{C}-\mathrm{V}$ measurements as a function of the deposition parameters

Fig. 7 DLTS signal at three different emission rates as a function of measurement temperature for MIS capacitor with a film grown at a pressure $400 \mathrm{mTorr}$ with gas Ratio-3

Fig. 8 Breakdown voltages versus pressure for different deposition routines

Fig. 9 SEM of the fabricated RF MEMS switch Design at $35^{\circ}$ tilt

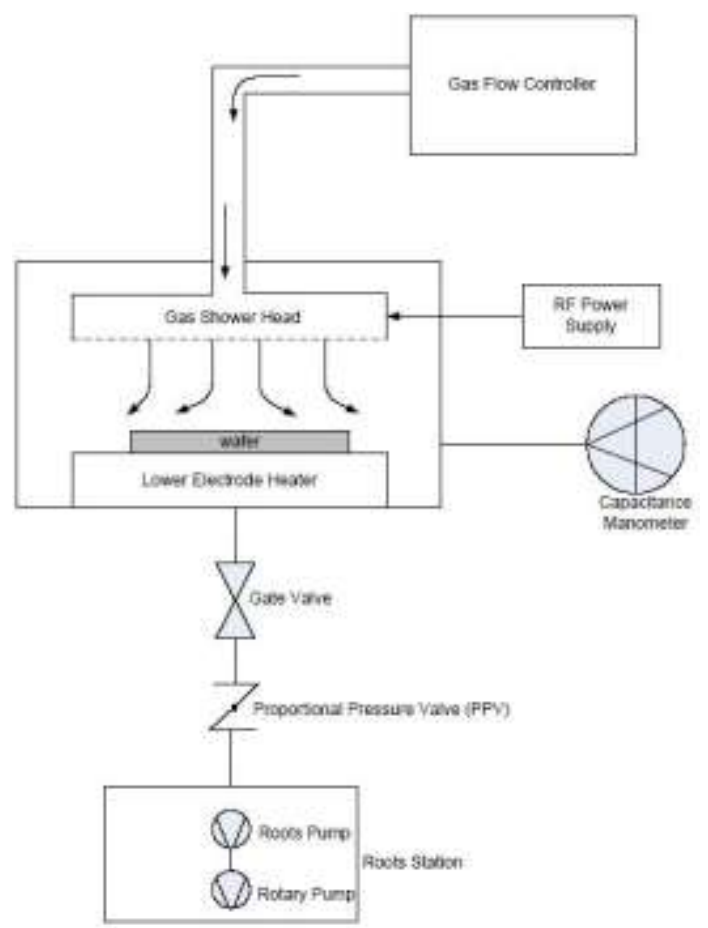

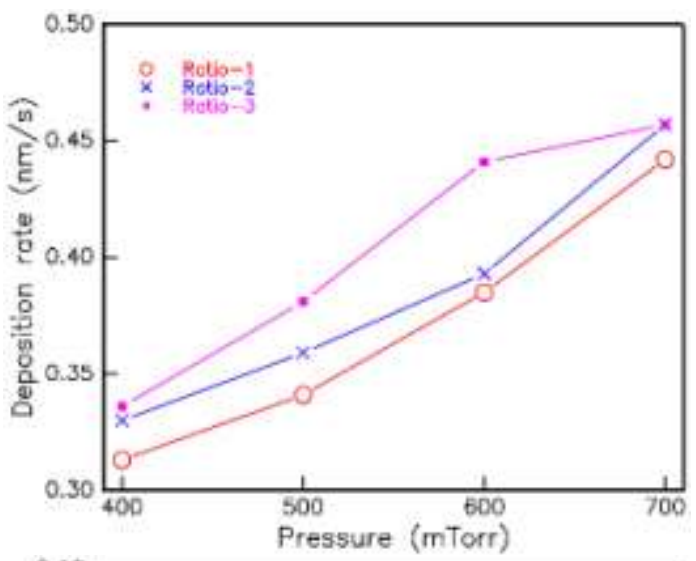
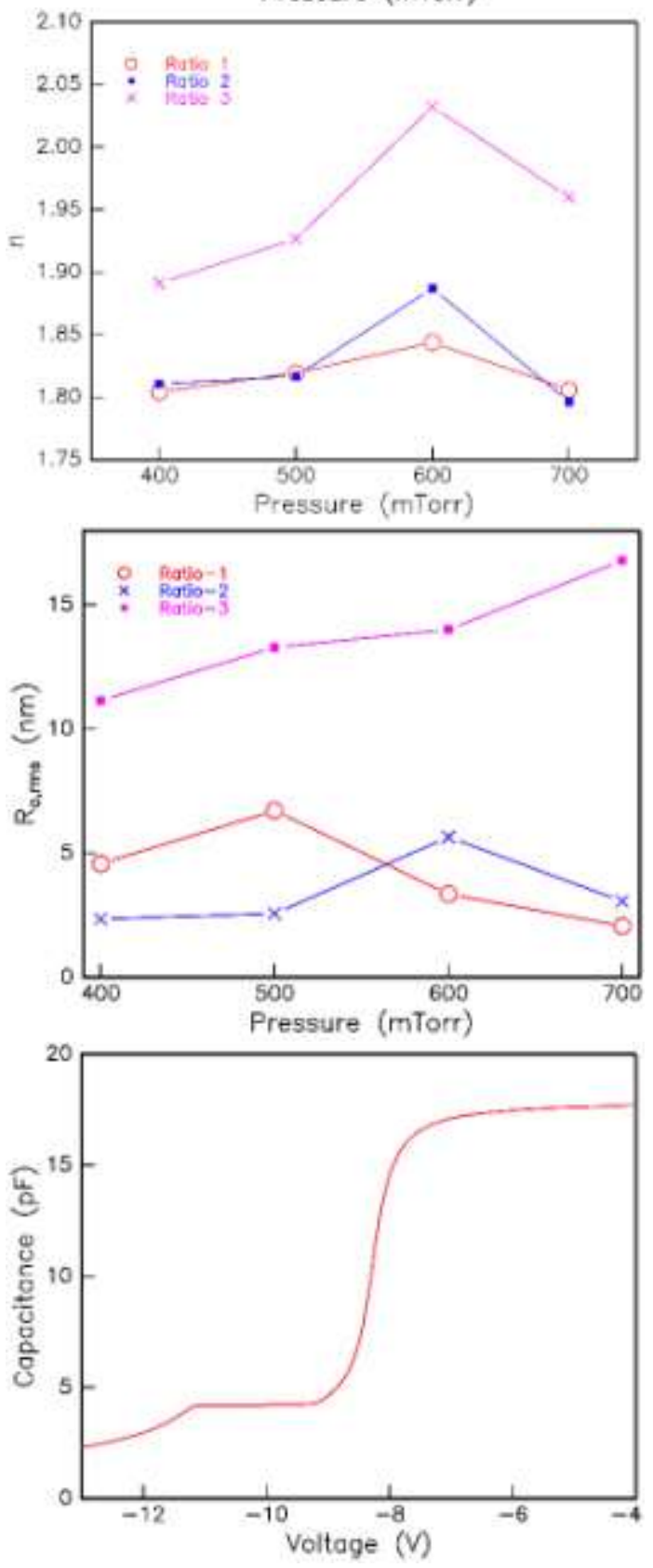

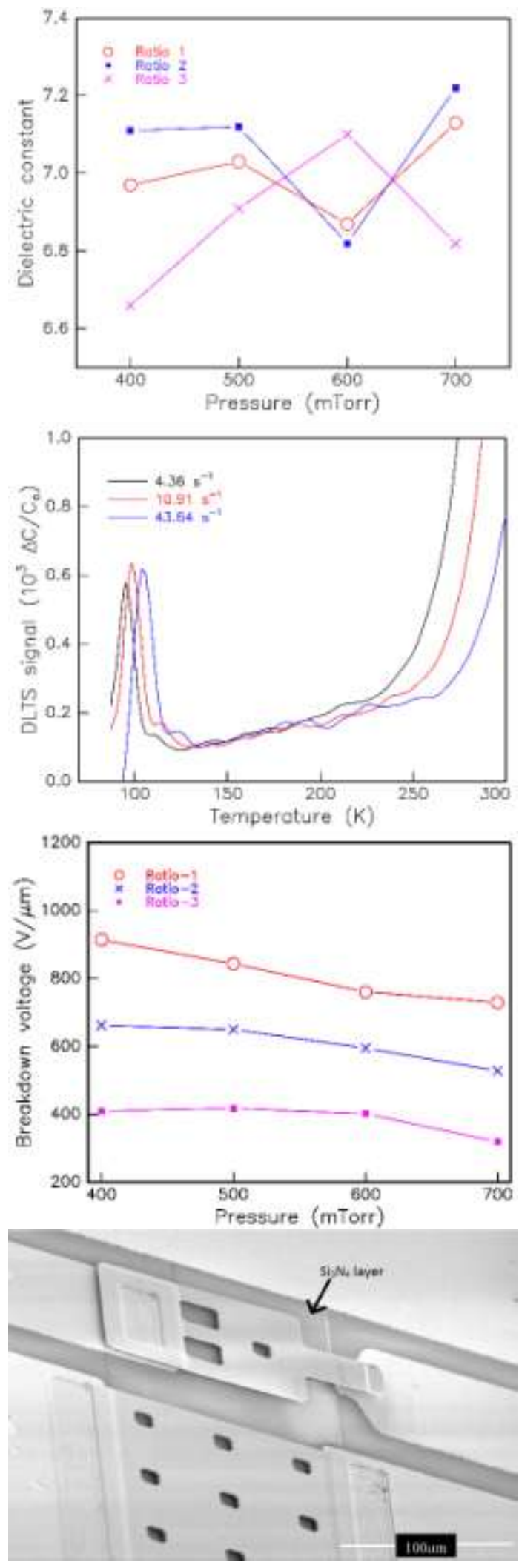


\section{University Library}

\section{- M M N E R VA A gateway to Melbourne's research publications}

Minerva Access is the Institutional Repository of The University of Melbourne

Author/s:

Rahman, HU;Johnson, BC;Mccallum, JC;Gauja, E;Ramer, R

Title:

Fabrication and characterization of PECVD silicon nitride for RF MEMS applications

Date:

2013-01-01

Citation:

Rahman, H. U., Johnson, B. C., Mccallum, J. C., Gauja, E. \& Ramer, R. (2013). Fabrication and characterization of PECVD silicon nitride for RF MEMS applications. MICROSYSTEM TECHNOLOGIES-MICRO-AND NANOSYSTEMS-INFORMATION STORAGE AND PROCESSING SYSTEMS, 19 (1), pp.131-136. https://doi.org/10.1007/s00542-012-1522-0.

Persistent Link:

http://hdl.handle.net/11343/283049 